\title{
Purulent Pericarditis: a Complication of Endoscopic Oesophageal Variceal Sclerotherapy
}

A 63-year-old woman with portal hypertension secondary to alcoholic cirrhosis underwent oesophageal variceal sclerotherapy with $5 \%$ ethanolamine oleate. She developed prolonged retrosternal pleuritic chest pain, with dyspnoea. Clinical examination revealed tachypnoea, tachycardia, and crepitations at the left lung base. Arterial blood gas analysis showed hypoxia of $7.7 \mathrm{~mm} \mathrm{Hg}$ (normal range $11.1-14.4 \mathrm{~mm} \mathrm{Hg}$ ), but the chest radiograph and electrocardiogram were normal. Since the D-dimer level was raised, a computed tomography (CT) pulmonary angiogram was carried out, which was negative for pulmonary embolism but showed pericardial and pleural effusions (Figure $\mathbf{1}$ ). The patient developed septicaemia with a temperature, tachycardia and hypotension, and had a raised white cell count and C-reactive protein level. Antibiotic treatment was started. She then developed signs of pericardial tamponade, with a raised jugulovenous pulse and muffled heart sounds. An echocardiogram showed a pericardial effusion $3.4 \mathrm{~cm}$ in size, with no features of tamponade. A therapeutic pericardiocentesis revealed purulent fluid, which grew coliform bacilli and Bacteroides species. A Gastrografin swallow on day 9 was normal. The patient underwent anterior thoracotomy with pericardiectomy, and made a slow but complete recovery.

There have been case reports of cardiac tamponade $[1,2]$ and mediastinal abscess [3] after variceal injection sclerotherapy, but not of patients requiring pericardiectomy in this situation. Autopsy studies have shown an incidence of oesophageal perforation of $2.9 \%$ [4]. This patient probably suffered an oesophageal perforation into the pericardial cavity due to a extravariceal injection of sclerosant, which formed a microabscess, resulting in purulent pericarditis. This was also the reason for the disparity between the clinical and echocardiographic features. Endoscopic variceal ligation is now the most effective treatment for oesophageal varices $(91 \%$; $95 \%$ confidence interval, $82.4-96.3 \%$ ) in

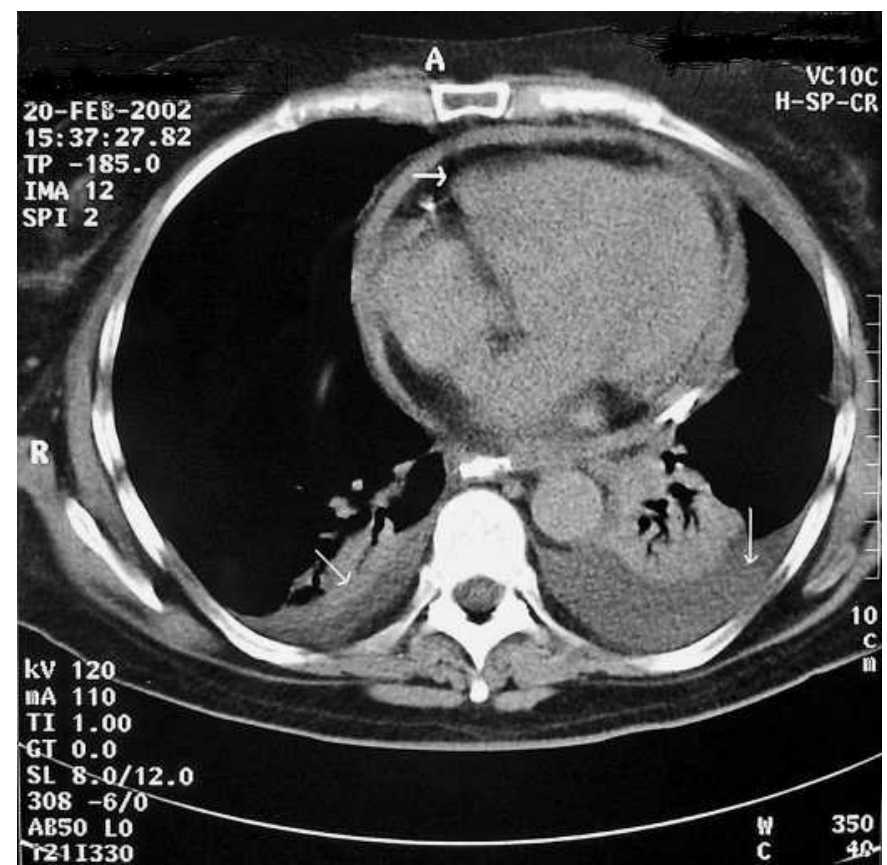

Figure 1 Computed tomogram of the chest, showing pericardial effusion (thick arrow) and bilateral pleural effusions (thin arrows), with basal atelectasis on the left side.

comparison to injection sclerotherapy (81.1\%; 95\% CI, 71.1-88.4\%) [5]. Oesophageal perforation resulting in purulent pericarditis is a potentially life-threatening complication after variceal sclerotherapy. Patients with prolonged chest pain following the procedure should be monitored for complications.

\section{S. Gupta, K. Thompson, L. Grellier}

Dept. of Gastroenterology,

Worthing Hospital, Worthing,

West Sussex, United Kingdom.

\section{References}

${ }^{1}$ Knaeur CM, Fogel MR. Pericarditis complication of esophageal sclerotherapy: a report of three cases. Gastroenterology 1987; 93: 287-290

2 Lam CS, Szeto ML. A rare complication of esophageal sclerotherapy. Hong Kong Med J 1997; 3: 101 - 103

${ }^{3}$ Althoff M, Schoenemann J, Weinhold ST et al. Mediastinal abscess following sclerotherapy of esophageal varices. Endoscopy 1995; 27: 630
${ }^{4}$ Korula J, Pandya K, Yamada S. Perforation of esophagus after endoscopic variceal sclerotherapy: incidence and clues to pathogenesis. Dig Dis Sci 1989; 34: 324-329

${ }^{5}$ Seewald S, Mendoza G, Seitz U et al. Variceal bleeding and portal hypertension: has there been progress in the last 12 months? Endoscopy 2003; 35: $136-144$

Corresponding Author

\section{S. Gupta, M.D.}

56 Sunningdale Road

Sutton SM1 2JS

United Kingdom

Fax: $\quad+44-208-7253520$

E-mail: san1gupta@hotmail.com 